\title{
BMJ Open Balancing nurses' workload in hospital wards: study protocol of developing a method to manage workload
}

\author{
W F J M van den Oetelaar, ${ }^{1} \mathrm{H}$ F van Stel, ${ }^{1} \mathrm{~W}$ van Rhenen, ${ }^{2,3} \mathrm{R}$ K Stellato, ${ }^{1}$ \\ W Grolman ${ }^{1}$
}

To cite: van den Oetelaar WFJM, van Stel HF, van Rhenen W, et al. Balancing nurses' workload in hospital wards: study protocol of developing a method to manage workload. BMJ Open 2016;6: e012148. doi:10.1136/ bmjopen-2016-012148

- Prepublication history for this paper is available online. To view these files please visit the journal online (http://dx.doi.org/10.1136/ bmjopen-2016-012148).

Received 11 April 2016 Revised 5 August 2016 Accepted 12 August 2016

CrossMark

\footnotetext{
${ }^{1}$ University Medical Center Utrecht, Utrecht, The Netherlands

${ }^{2}$ Center for Human Resource Organization and Management Effectiveness, Business University Nyenrode, Breukelen, The Netherlands

${ }^{3}$ ArboNed Occupational Health Service, Utrecht, The Netherlands
}

Correspondence to WFJM van den Oetelaar; W.f.j.m.vandenoetelaar@ umcutrecht.nl

\section{ABSTRACT}

Introduction: Hospitals pursue different goals at the same time: excellent service to their patients, good quality care, operational excellence, retaining employees. This requires a good balance between patient needs and nursing staff. One way to ensure a proper fit between patient needs and nursing staff is to work with a workload management method. In our view, a nursing workload management method needs to have the following characteristics: easy to interpret; limited additional registration; applicable to different types of hospital wards; supported by nurses; covers all activities of nurses and suitable for prospective planning of nursing staff. At present, no such method is available.

Methods/analysis: The research follows several steps to come to a workload management method for staff nurses. First, a list of patient characteristics relevant to care time will be composed by performing a Delphi study among staff nurses. Next, a time study of nurses' activities will be carried out. The 2 can be combined to estimate care time per patient group and estimate the time nurses spend on non-patient-related activities. These 2 estimates can be combined and compared with available nursing resources: this gives an estimate of nurses' workload. The research will take place in an academic hospital in the Netherlands. 6 surgical wards will be included, capacity $15-30$ beds. Ethical considerations: The study protocol was submitted to the Medical Ethical Review Board of the University Medical Center (UMC) Utrecht and received a positive advice, protocol number 14-165/C.

Discussion: This method will be developed in close cooperation with staff nurses and ward management. The strong involvement of the end users will contribute to a broader support of the results. The method we will develop may also be useful for planning purposes; this is a strong advantage compared with existing methods, which tend to focus on retrospective analysis.

\section{INTRODUCTION}

Hospital management is exploring ways to ensure a good balance between patient needs and nursing staff size and expertise in order to deliver good quality care and

\section{Strengths and limitations of this study}

- Instead of classifying patients in categories of nursing intensity, patient characteristics are related to their actual care time.

- Covers all activities of nurses, not just direct care time.

- Combines multiple dimensions of nursing workload in one study.

- Statistics: the mixed model corrects for multilevel data.

- Setting in the surgical wards of an academic hospital only.

excellent service to their patients while managing operational excellence. At the moment, it is challenging to objectively determine whether nursing capacity is optimally matched to patient needs on the hospital wards. We aim for a fair and sensible distribution of nursing staff over the wards, resulting in an equally distributed and manageable workload for all nursing staff. This requires a good fit between patients' needs and nursing staff. One way to ensure a proper fit is to work with a workload management method for nursing staff. In theory, this should help balance required resources with available resources, which prevents extra costs for overstaffing a nursing ward and, on the other hand, prevents a decline in patient experiences or employee engagement by understaffing a ward. There is a direct relationship between nurses' workload and patient outcomes ${ }^{1-4}$ and nurse-reported quality of care. ${ }^{5}$ Good workload management will also help keep employees healthy, as high workload is a predictor for burnout ${ }^{6} 7$ and absenteeism. Bakker $^{9}$ found a relation between job demands such as workload and performance. In their systematic literature review, Toh $e t a l^{10}$ found a positive bidirectional 
relation between the nursing shortage and oncology registered nurses' job dissatisfaction, stress and burnout.

In addition, nursing staff is relatively scarce in the Netherlands and it is not expected that this will change in the near future. Labour demand is expected to increase as the population ages, with consequences for future skills and competences while the number of health professionals decreases, resulting in healthcare labour shortages. ${ }^{11}$ Workload has been shown to have an effect on nurses' intention to leave ${ }^{12} 13$ and on job outcomes, ${ }^{14}$ both directly and as a mediating factor. High turnover of nursing staff results in higher costs for training of new nurses or using temporary staff ${ }^{315}$ and therefore needs to be minimised.

There is extensive literature that describes the workload of nurses. Workload is often not clearly defined and is usually measured by asking nurses to fill out questionnaires about perceived workload, mostly one dimension of workload such as mental load or amount of work. ${ }^{16}$ Holden et $a l^{17}$ describe three different dimensions of workload: task-level, job-level and unit-level workloads. These workload types describe different dimensions of workload and each type of workload has an effect on burnout, job dissatisfaction and medication errors likelihood. In their article, Holden et al recommend also taking emotional and physical load into account.

Many studies have identified factors that predict the workload of nurses. There is evidence that these nursepatient ratios or nursing hours per patient day (NHPPD) do not accurately predict workload or nurses, ${ }^{18}$ since these do not take into account the different needs between patients nor the differences in experience and education level of nursing staff. Twigg and Duffield ${ }^{15}$ argue that relying on expert opinion in setting standards for workload, in their study a standard NHPPD per ward, is not optimal and recommends using a standardised patient acuity measurement.

In other methods, workload is predicted by quantifying the effect of patient characteristics or characteristics of the treatment on workload. Mueller $e a^{19}$ tested the correlation between the Barthel index scores and acute International Classification of Functions (ICF) core sets and nurses' workload. In this research, 20-44\% of perceived nurses' workload variance is explained by these scores. This indicates that patient characteristics matter in nurses' workload. This research was performed in a critical care setting and has not yet been replicated in general hospital wards or other environments. In Belgium, hospitals are required to register the Belgium Nursing Minimum Data Set (B-NMDS) in order to benchmark hospitals on several dimensions, among which workload. Van den Heede $e t a l^{20}$ show that $70 \%$ of variation in nursing staff per unit is predicted by the B-NMDS item hospital type with the covariates nursing intensity and service type. They recommend that instead of working with NHPPD, an NHPPD corrected for nursing intensity is a better measure. However, Sermeus et $a l^{21}$ stated in a 2008 study that the B-NMDS nursing intensity did not necessarily give an indication of required nursing time. Another drawback of the B-NMDS is the extensive amount of registration required by the hospitals. ${ }^{22}$

Hughes $e t a l^{23}$ found that correcting the standard midnight census measure for patient churn gives a better indication of nurses' workload. Myny et $a l^{22}$ determined a set of 28 measurable factors that are expected to influence the workload of nurses, of which 3 are recommended for incorporation in a workload management methods: the number of work interruptions, the patient turnover rate and the number of mandatory registrations. It is noted that Myny et al performed their research in Belgium, where hospitals are required by law to participate in the B-NMDS, which would explain the perceived high importance of registration on workload.

Several workload management methods are found in the literature. The RAFAELA patient classification system $^{24}$ is an instrument to assess optimum levels of nursing intensity. We consider this as a form of workload management. The RAFAELA system consists of the Oulu Patient Classification instrument, ${ }^{25}$ a system that records daily nursing resources, and the Professional Assessment of Optimal Nursing Care Intensity Level questionnaire. The three are combined to measure nursing intensity. RAFAELA measures only the patient-related workload of nurses and does not include other tasks. ${ }^{26}$ This method is widely used in Finland; while promising, it is not used for prospective workload management but only for assessments of workload in the past. For optimal versatility of nursing staff, prospective insight is of great value.

Hoi et $a l^{27}$ developed a workload intensity management system by defining 28 relevant nursing diagnoses and performing a work sampling study on nurses' activities. A nursing time per day was identified for each diagnosis, and for each ward the significant nursing diagnoses were determined. Hoi et al developed a prediction model with a fixed component of nursing time for each patient admitted to a ward, a fixed nursing time for each occurrence of a diagnosis and a fixed time for indirect patient care. Depending on the number of patients and the patient mix, a forecast of required nursing time could be made. In this study, $60-70 \%$ of variance in nursing time was explained by these nursing diagnoses. Hoi et al also found that his patient dependency measurements were not correlated with nursing time.

Some aspects that influence workload fall outside the scope of the current project. Some research has examined the relation between nurses' workload and unit-related characteristics such as ward lavout and number of single rooms in a ward. ${ }^{22}$ Since we cannot influence these factors without major renovations, we chose not to include them in our research. Furthermore, some studies focus on the relation between nurses' perceived workload and job resources such as support from 
colleagues or ward management or relationship with medical staff. ${ }^{16}{ }^{16}$ We chose not to focus on this domain at the moment. We are aiming for a fair distribution of work between our wards, regardless of ward-specific job resources that can counter the job demand workload.

Workload is dependent on the amount of work that is given to the staff, as well as on the resources available to handle this amount of work. There is literature that suggests that a higher proportion of registered nurses in the nursing staff results in lower workload and better patient outcomes, ${ }^{28}{ }^{29}$ but there is no research that quantifies differences in proficiency in nursing staff: what are the required nursing resources when we account for numbers of students of different levels and experience of registered nurses?

Some countries such as Australia seem to have commercial packages that manage workload, but there is no scientific evidence to support their effectiveness and these packages are not available in the Netherlands.

Our goal is to find a better match between required and allocated nursing staff, under the condition of at least the same levels of patients' experiences and nurses' engagement. With this study, we aim to contribute to developing a workload management method that is user-friendly (easy to interpret and requiring limited additional registration); that is applicable to different types of hospital wards; that will differentiate between different levels of nurses' proficiency; and that is endorsed by nurses and nurse management and covers all activities of nurses (not just those activities that are directly patient-related). In a later phase, we will study whether the method is suitable for prospective planning of nursing staff.

This study protocol describes the steps we will take to develop a new workload management method and suggests a method to test its validity.

\section{METHODS}

\section{Workload management method}

The University Medical Center (UMC) Utrecht has experience with a workload management method developed by the former Dutch National Hospital Institute, $\mathrm{NZi}^{30}$ We use the framework of this methodology as a starting point to develop our own workload management method, because it already meets many of the requirements stated previously. The NZi methodology consisted of the following items:

1. A checklist of nine patient characteristics that lead to a classification of light, moderate, heavy and intensive care;

2. Time study of nurses' activities, registering time spent on direct and indirect patient care, unit-related tasks and other tasks;

3. Estimate of allocated nursing resources;

4. Questionnaire of perceived workload and perceived quality of work.
These data are combined to estimate and validate the workload of nurses.

The NZi method has several advantages: it is easy to use, does not require much additional registration and can be used in a wide variety of hospital wards. However, the method also has some drawbacks. Nurses feel that important factors that influence care time are missing in the patient classification, such as isolation measures and psychosocial care. They often feel that after classification of a patient, the resulting class does not reflect the actual workload. Also, the patient classification is rather crude: it consists of four categories (intensive, intermediate, moderate, light) of which only three occur on regular hospital wards. In addition to this, the method does not differentiate for levels of nursing experience; student nurses are obviously not as efficient as experienced, certified nurses. Finally, the checklist used to measure nurses' perceived workload is not validated. Owing to these disadvantages, the method was not supported by the nursing staff and was eventually discontinued.

We will use the NZi workload management method framework as a basis for our development though, since it has potential to fulfil the requirements we stated earlier, but will make several adjustments to correct for the aforementioned drawbacks. Since the patient classification in this method is neither evidence-based nor widely supported by nurses, we will use a new list of patient characteristics expected to influence care time. Also, we will more specifically determine the required nurse resources, differentiating for levels of education and experience. Finally, we plan to use a validated questionnaire to determine nurses' perceived workload. We choose to measure five dimensions of perceived workload: work pace (time pressure), amount of work, emotional load, physical load and mental load, as experienced by nurses. This will result in the following adjusted approach (figure 1).

The research will take place in an academic hospital in the Netherlands. Six surgical wards will be included, ward capacity varying from 15 to 30 beds (2 wards with 15 beds, 4 wards with 30 beds). We will focus on nurses' workload in the day shift. Workload of other types of ward staff (doctors, assistants, cleaners, etc) will not be considered in this study. In the following paragraphs, we give an extensive explanation of the procedural steps for setting up the workload management method described in figure 1 .

\section{Identifying relevant patient characteristics}

We decided that we do not want to classify patients in categories of intensity of care, but prefer to directly predict care time of patient characteristics. The composition of a list of patient characteristics relevant to care time will be carried out in a Delphi study consisting of five phases. In this study, we will identify relevant patient characteristics for the specific setting of our study (six surgical wards). If successful, we can extend this method 


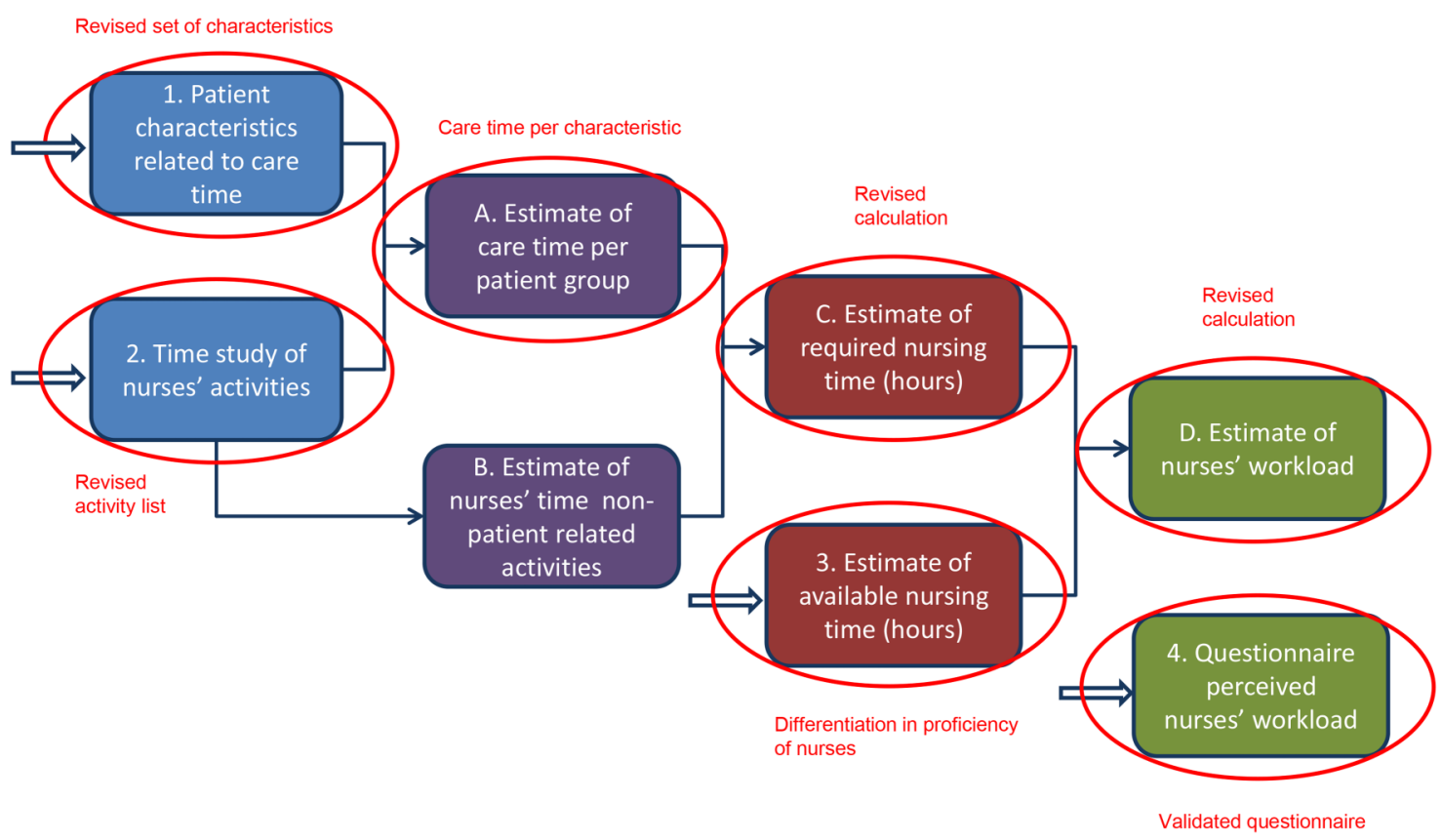

Figure 1 Components of new workload management method.

to a larger setting in a later phase. We choose the Delphi method in order to acquire an expert opinion on relevant characteristics. ${ }^{31}$ Representatives from all six wards will take part in Delphi rounds to determine patient characteristics that, in their opinion, have the most influence on care time and thus on nurses' workload. All participants should be experienced senior nurses or nurse team leaders, with one representative from each ward. All participants will receive extensive information about the purpose of the research and how the Delphi study contributes to it. The central question will be: Which patient characteristics cause nurses to spend more time caring for a patient? When a patient is admitted to a ward, nurses will always spend a certain amount of care time for this patient, regardless of the reason for admission. For example, time that is spent on handing out meals, having a chat or tidying up. On top of this 'baseline' care time, nurses spend time catering to specific needs for a particular patient, based on characteristics of that patient (eg, the procedure the patient has undergone). We assume that there is always a baseline amount of care that is provided to a patient when admitted to a ward, as also suggested by Hoi et $a l^{27}$ This baseline care time will not be defined as beforehand, because what is considered baseline care may differ from ward to ward. We will let the study results define the baseline care time per ward. Our study will focus on finding patient characteristics that are expected to cause additional care time, on top of this baseline care time.

\section{Phase 1: Delphi group composition and interviews}

Based on separate interviews with all six Delphi group members, a starting set of characteristics, with corresponding definitions, will be composed. Clear definitions of characteristics are necessary to make sure they will be uniformly interpreted and do not overlap. Results will be shared and the Delphi group will comment on the clarity of the definitions. Definitions will be refined and results shared.

\section{Phase 2: prioritisation}

In the next phase, the Delphi group will be asked to prioritise the characteristics by dividing a fixed number of points over the characteristics. The group will be free to divide the points as they see fit, so they can allocate 0 points to a characteristic if they consider it to be irrelevant, or all points if they consider the characteristic as the only relevant one on the list. Perceived importance of characteristics is expected to vary per ward. Characteristics with close to 0 points total score will be removed from the checklist. Results will be shared in the group and combined in a checklist. In the preparation of the next phase, a test version of this checklist of patient characteristics will be implemented in the hospital information system. This checklist will consist of all identified patient characteristics, with a yes or no tick box beside each characteristic. For each characteristic, the full definition will be visible.

\section{Phase 3: preliminary testing}

Nine new members will be added to the Delphi group, all senior staff nurses or team leaders. The whole Delphi group will be trained in how to use the new digital checklist. They will be asked to fill out the checklist for 10 random patients admitted on their wards. These will be the first 10 patients on their ward overviews of one particular day (to be selected). After this test, the Delphi 
group members will be interviewed and asked if the test version of the checklist is complete, if the definitions are sufficiently clear and if characteristics are mutually exclusive. They will also be asked if the checklist is not too time-consuming: Is the administrative burden of filling this out for every patient every day reasonable? Based on this test period, definitions will be refined and the new version of the checklist will be uploaded in the hospital information system.

\section{Phase 4: extensive testing}

After the preliminary testing, a new, extensive test period will start. All wards will select nurses to the daily task of filling out the checklist of patient characteristics for all patients on the ward. These nurses will all be uniformly trained in how to use the checklist. The training will consist of information on the purpose of the research and how the filling out of the checklist contributes to the research. The importance of accuracy will be stressed and nurses will be informed that checklist input will also be monitored by random checks. This is done in order to detect mistakes early, as well as to avoid manipulation of input. The process of development of the checklist will be explained and each characteristic and definition will be clarified. Each nurse will be personally instructed and a short guidebook will be available next to every computer on every ward. For a period of 1 month, checklists will be filled out every day shift for every patient on every ward participating in the study. A medical student will be trained in how to use the checklist as well. This student will retrospectively check the registrations in 40 randomly selected checklists filled out by the nurses during the test period. At the end of this test period, the Delphi group members will be interviewed again and, where necessary, changes will be made and definitions will be sharpened.

\section{Phase 5: implementation}

Test results will be processed and a final version of the checklist will be composed and entered in the hospital information system. All involved nurses will be informed of the changes and will receive new working instructions. A reporting tool will be developed to track actual usage of the checklist. During the time study period (see 'Time study nursing staff' section), this reporting tool will be used to make sure a checklist is filled out daily for each patient present in the day shift on each ward.

\section{Time study nursing staff}

In order to determine whether the aforementioned patient characteristics indeed affect care time, a work sampling study will be carried out. Work sampling results in a random sample of the activities of nurses and is a useful and cost-effective methodology to explore work-related activities. ${ }^{32}$ From this study, we expect to gain broad insight into the way nurses spend their working hours, and to what extent their work is directly patient-related. Ampt $e t a l^{33}$ suggested working with trained observers as an alternative to self-reporting, because the latter can be prone to bias. This is only possible when the staff to be observed is in an area that can be overseen by the observer and the latter can determine the activities relatively easily. For example, if work sampling is carried out on staff that is moving great distances or is performing mostly cognitive tasks, then selfreporting can be better. They also advocate the use of handheld computers to make registration faster and more accurate.

Sittig $^{34}$ gave important tips when designing a work sampling study in healthcare: involve the nurses and nurse management in the study, determine relevant activities to register and make foolproof definitions, identify the right observers and train them well, and do pilot samples to test the set-up.

The same six surgical wards aforementioned will participate in this study. The study will focus on activities of nurses in the day shift. Weekends will be excluded because task mix and staffing is very different on weekends and cannot be compared with day shifts of regular weekdays. Team leaders and students will be included in the study. Ward managers will be excluded because they are not active in direct patient care.

A set of activity groups will be identified as the relevant activities to register. For each activity group, we will describe which activities are related to these groups. The activity groups will be grouped into four categories: direct patient care, collective patient care, general tasks and other tasks. Direct patient care is defined as care that can be directly related to one specific patient. This includes 12 activity groups, with activities among others including assistance with bathing or eating, handing out medication, changing bed linen, wound care, and communication with patient or family. Collective patient care is defined as tasks that are patient-related, but are impossible to attribute to a specific patient. This includes four activity groups, with activities among others including general preparation of medication, patient handover and bringing a collection of samples to the laboratory. General tasks include five activity groups, with such activities as education, meetings, organisation of work (planning), administrative duties and domestic duties. Other tasks include three activity groups, with activities such as lunch and coffee breaks and personal time. This set of activities will be determined by the same group of nurses in the Delphi group who will also determine relevant patient characteristics. There will only be one round of evaluation since we do not expect much disagreement.

During the time study, all nurses in the day shift will be observed approximately every $10 \mathrm{~min}$. Trained observers will register activities approximately every $10 \mathrm{~min}$ in the day shift, starting at 7:30 and finishing at 16:00. Observations will be registered on a handheld computer. Exact start and finish times will depend on the random 
time interval generator of the handheld computer. Time intervals will be automatically randomised between 8 and $13 \mathrm{~min}$, with an average of 10 minutes. Observers will be asked to register three things each time they make an observation: the name of the nurse, the activity the nurse is performing and, when the activity is patientrelated, the details of the patient concerned. In this way, a random survey of nurses' activities in day shifts will be carried out.

First, a test study will be performed. The aims of this test study are as follows:

- To test the handheld computer equipment and its accessories: do they work properly and are they easy to use?

- To test the activities list: is it complete and easy to interpret?

- To test the workload of the observers: how many nurses can be observed by one observer?

Subsequently, the actual work sampling study will be planned. A representative time period will be carefully selected. The study needs to be planned in a period in which workload is expected to be average; outside holiday seasons, periods with especially high or low occupancy rates (eg, due to reduction of operating room capacity) or periods with enhanced or reduced nursing capacity (eg, due to planned education). Also, the number of observations in the work sampling study needs to be sufficiently large. For practical reasons, UMC Utrecht wants to limit the work sampling study to 3 weeks, or 15 day shifts. The main practical obstacles are that we will not be able to find and train enough observers to cover a longer time period and the high costs of extending the duration of the study. A preliminary question round with ward nurses indicates that we can expect to find a maximum of 15-20 patient characteristics. The participating wards have an average of 25 patients admitted per ward at any given time. In this context, one observation is the measurement of care time for one patient during a 1-day shift. This means a total of $150(6 \times 25)$ observations per day shift. The maximum of 3 weeks (15 working days) of study time period then limits us to a maximum of $15 \times 150=2250$ observations for each patient characteristic. Since we do not know which characteristics we will identify as relevant, it is not possible to estimate what the chances are that this characteristic will be observed sufficiently often during a 15-day time period. When sampling nurses' activities every $10 \mathrm{~min}$, we will generate $\sim 54000$ observations ( $=15$ study days $\times 6$ wards $\times 12$ nurses per $\operatorname{ward} \times 50$ observation rounds per day shift) of nurse activities. On the basis of a previous time study of 10 day shifts in 2003 on three of the same wards at UMC Utrecht, we estimate that we will define between 25 and 30 activities. This 2003 study worked with 23 activities, which are all still relevant today. We do miss items such as handover though, so we assume we will determine a few more activities in this study. The database from the 2003 study is lost, but the reports on the overall results are still available. From the 2003 time study, we know that the most observed activity accounted for $\pm 9 \%$ of observations in 2003. This translates to a maximum of \pm 4.860 observations per activity in our study.

Observers will be selected and uniformly trained in how to register nurses' activities. Observers will be either nurses from wards in the study (observing other wards than their own) or medical students. We prefer to work with nurses as observers where possible, because they are motivated to register activities accurately and they are familiar with the activities and therefore less likely to misinterpret or make mistakes. As a bonus, nurses learn about working procedures on other wards besides their own, which broadens their horizon and will help exchange ideas and understanding between wards.

During the work sampling, observers will register two or three variables with each observation: name of the observed nurse, activity and, when applicable, the related patient. All are categorical variables with many possible categories to choose from: up to 15 names of nurses, up to $20-30$ activities and up to 30 patients. For an accurate end result, it is important that all three variables are registered correctly. To test the reliability of the registrations, an inter-rater agreement test will be planned during the 3-week time study period during which the work sampling will take place. For this test, a second observer will temporarily join the scheduled observer. Both observers will have had the same training and both will have already done at least one shift of observing during the time study. Regular tests for interrater agreement, such as Cohen's $\kappa$ or intraclass correlation, cannot be applied here because these assume that only one variable is observed and $\kappa$ also assumes that a variable is classified in a limited (ie, maximum of four) number of categories. ${ }^{35}$ Therefore, we will determine inter-rater agreement as the percentage of exact agreement on all three variables.

\section{Estimating required care time}

'Time study nursing staff' section described how data will be gathered. In this section, we describe how we plan to analyse the data in order to derive required care time.

Results of the patient characteristic checklist will be combined with work sampling results. Data will be analysed from the perspective of the nurse (How do they spend their time?) and the perspective of the patient (How much time is spent on caring for patients?). When analysing from the patient perspective, we will combine information on patient characteristics and care time per patient per date. In this way, it is possible to analyse the relation between patient characteristics and care time: Does care time increase when certain characteristics apply? Also, what is the baseline care time for a patient when none of the characteristics apply?

For the analysis of care time per patient, we will use linear mixed-effects models. ${ }^{36}$ These models will be used to determine the significance of the characteristics in 
relation to care time and to estimate the additional care time per significant characteristic. This method has not been used before in studies in this field. We choose linear mixed-effects models because we will be working with multilevel data: care time will be measured more than once for most patients, since the majority will be admitted for more than 1 day. We therefore wish to explain variability of care time within the length of stay of a patient, as well as variability of care time between patients. In the mixed models, the ward and patient characteristics will be taken as fixed effects (since we are specifically interested in analysing the effects of these). A random intercept, and potentially a random slope for time, per patient will be included in the models to adjust for clustering of measurements within patients.

Results of this analysis can be used for planning purposes: we plan to register the significant patient characteristics continuously and fill a database with the results. This database can be used to define patient profiles (linked to diagnoses or treatment types), where a profile of expected patient characteristics per treatment day can be determined. These profiles can be regularly updated and used for planning purposes, for example, when linked to the OR schedule, to predict expected workload in the future. An example of such a profile can be found in table 1 .

As stated above, we assume that there is a 'baseline' care time: an amount of time that is spent on caring for a patient when none of the aforementioned characteristics apply. We will derive estimated means from the linear mixed-effects model for a patient profile in which none of the characteristics are present. In this way, we can estimate this baseline care time.

Nurses also spend time on other activities that are not directly patient-related, such as household tasks, administration, taking care of supplies, training students, etc. Per ward, we will determine the percentage of time that nurses spend on tasks that are not directly patient-related from the work sampling results. These estimated percentages will added to the estimated time for patient-related tasks, so that the total required nursing resources (expressed as care time) can be calculated.

\section{Estimating allocated care time}

Allocated care time can be calculated by simply counting the number of nurses in a shift and multiplying this amount by the shift hours. However, there is evidence that indicates that a staff mix with a large proportion of registered nurses results in better quality of care and a better managed workload. ${ }^{28}{ }^{29}$ Therefore, it is necessary to introduce nurse education levels into the workload equation. However, besides this, we believe that working experience of nurses is also a factor of importance; nurses' experience is related to clinical expertise. ${ }^{37}$ There have not been any studies to relate clinical expertise to perceived workload, but we believe that a more experienced nurse is more likely to handle workload better than a relatively newly registered nurse. Our hospital works with different types of student nurses. Dependent on the type of education these students are following and the study year they are in, they are more or less proficient; depending on their proficiency, they can be allocated to more or less different tasks. In order to capture all these types of differences in nursing staff, we introduce a proficiency percentage. A fully registered nurse who has more than 1 year experience on the ward he or she is allocated to is set to a proficiency percentage of $100 \%$. These nurses are qualified to perform all the different tasks in the unit and have sufficient experience to be proficient in them. If the nurses are not fully trained yet, or have not been working in the specific specialty for a long time, then they are likely to be less proficient than nurses who have. We will ask ward management (head nurses) for their expert opinion to define a proficiency per cent of all less experienced nurses $(<1$ year experience on the ward they work in) and student nurses (for two education types known in the Netherlands and the status of their educationsenior or junior students). Management is asked to determine the proficiency of this type of nurses against the $100 \%$ standard. Determination of proficiency for individual nurses in the study will be carried out by classifying nurses in this framework. We will translate this proficiency into allocated care time: a nurse in an 8-hour shift with $100 \%$ proficiency will represent allocated care time of 8 hours. A nurse with $75 \%$ proficiency will represent 6 hours of allocated care time in an 8-hour shift.

\section{Estimating nurses' workload}

An estimate of nurses' workload can be made by dividing the estimate of allocated care time by the estimate of required care time.

Table 2 displays our plan for processing the aforementioned components into a workload management method.

Table 1 Workload profile for patient group $\mathrm{X}$ (fictitious example)

\begin{tabular}{|c|c|c|c|c|c|c|}
\hline Patient characteristic/day of stay & Care time for characteristic & Day 1 & Day 2 & Day 3 & Day 4 & Day 5 \\
\hline Characteristic 1 & $20 \mathrm{~min}$ & $x$ & & & & \\
\hline Characteristic 2 & $10 \mathrm{~min}$ & $\mathrm{x}$ & $x$ & $x$ & & \\
\hline Characteristic 3 & $15 \min$ & & & $x$ & $x$ & \\
\hline Characteristic 4 & $15 \min$ & & & & & $x$ \\
\hline Characteristic 5 & $5 \mathrm{~min}$ & & & & & $x$ \\
\hline Total care time per day & & 30 & 10 & 25 & 15 & 20 \\
\hline
\end{tabular}


Table 2 Workload management method (fictitious example)

\begin{tabular}{|c|c|c|c|c|}
\hline Ward $X Y Z$ & June 4 & June 5 & June 6 & \\
\hline Care time for direct patient care (hours) & 46 & 39 & 46 & \\
\hline Time for indirect patient care and additional tasks (hours) & 48 & 40 & 48 & \\
\hline Outcome C: total required care time (hours) & 94 & 79 & 94 & \\
\hline Nurse qualifications & Proficiency & $\begin{array}{l}\text { Allocated care } \\
\text { time (hours) }\end{array}$ & $\begin{array}{l}\text { Allocated care } \\
\text { time (hours) }\end{array}$ & $\begin{array}{l}\text { Allocated care } \\
\text { time (hours) }\end{array}$ \\
\hline Registered nurse $\geq 1$ year experience & $100 \%$ & 10 & 8 & 9 \\
\hline Registered nurse $<1$ year experience & $85 \%$ & 0 & 1 & 1 \\
\hline Student nurse senior & $70 \%$ & 2 & 0 & 2 \\
\hline Student nurse junior & $45 \%$ & 1 & 0 & 0 \\
\hline Outcome 3: total allocated care time (hours) & & 95 & 71 & 90 \\
\hline \multicolumn{2}{|c|}{$\begin{array}{l}\text { Outcome D: expected per cent overstaffing or understaffing } \\
\text { (workload indication) }\end{array}$} & $1 \%$ & $-11 \%$ & $-4 \%$ \\
\hline \multicolumn{2}{|l|}{ Outcome 4: average perceived workload (scale of 1 to 5 ) } & 3,1 & 4,2 & 3,2 \\
\hline
\end{tabular}

Patient type profiles for all admitted patients in a shift can be added up to get to the total required care time for patient-related activities (A) for that shift.

From the time study, an estimate for time spent on non-patient-related activities (B) per shift can also be made. Together, derived components $\mathrm{A}$ and $\mathrm{B}$ can be combined to determine the estimated required nursing time $(\mathrm{C})$. The allocated nursing time (3) is determined by counting the number of nurses on duty and multiplying this by the shift time. This will be performed for each type of nurse on duty (registered, student, etc).

Dividing the allocated nursing time (3) by the required time $(\mathrm{C})$ gives an indication of nurses' workload (D): $0 \%$ is assumed to be a perfect fit, positive numbers indicate overstaffing and negative numbers understaffing. This indication will then be compared with the perceived workload (4) from questionnaires answered by nurses on duty during that shift.

When this workload management method is used, the only registration that has to be made on a daily basis is the patient classification, which should only take a few minutes per day per ward.

\section{Measuring perceived nurses' workload}

Job demands and resources will be assessed with shortened scales ${ }^{38}$ of the validated Questionnaire on the Experience and Evaluation of Work (QEEW), which is widely used by Dutch occupational health services and applied researchers. ${ }^{39} 40$

The QEEW has been validated for determining engagement and related resources (such as support from colleagues) and related demands (such as workload). It will be measured once as a baseline measure for engagement of nurses. The questionnaire contains 98 questions, so it is not practical to use it for measurements on a daily basis. Therefore, we have selected 12 questions to measure outcomes such as stress level and engagement, as well as resources and demands on a daily basis. Of these 12 questions, 5 consider different demands related to workload (pace and amount of work, emotional load, physical effort and mental load). The following table shows these five questions, measured on a five-point response scale (table 3).

Every answer option corresponds to a certain weight (points ranging from 1 to 5 for the answers 'not at all' to 'all the time'). We will test the internal consistency of the workload items by calculating Cronbach's $\alpha$. We will also test what happens to Cronbach's $\alpha$ if one of the items is deleted from the questionnaire. We expect that every question has an equal weight in measuring workload.

The shortened 12-item questionnaire will be filled out every day during the work sampling period by each nurse on duty, at the end of the day shift. Per ward,

Table 3 Questionnaire perceived workload

\begin{tabular}{lllllll}
\hline $\mathbf{N}$ & Question & Answer & & & \\
\cline { 2 - 6 } & Not at all & Sometimes & Regularly & Often & All the time \\
\hline 1 & Did you have to work very fast today? & 0 & 0 & 0 & 0 & 0 \\
2 & Did you have too much work to do today? & 0 & $O$ & $O$ & $O$ & 0 \\
3 & Did you consider your work mentally very challenging today? & $\mathrm{O}$ & $\mathrm{O}$ & $\mathrm{O}$ & $\mathrm{O}$ & $\mathrm{O}$ \\
4 & Did your work demand a lot from you emotionally today? & $\mathrm{O}$ & $\mathrm{O}$ & $\mathrm{O}$ & $\mathrm{O}$ & $\mathrm{O}$ \\
5 & Did you find your work physically strenuous today? & $\mathrm{O}$ & $\mathrm{O}$ & $\mathrm{O}$ & $\mathrm{O}$ & $\mathrm{O}$ \\
\hline
\end{tabular}


individual scores of workload are added up and averaged for all nurses in the day shift on that ward. The shortened questionnaire will be validated against the QEEW.

\section{Validation}

We plan to validate the workload management method by comparing the estimated nurses' workload to the workload as it was perceived by the nurses on duty (table 4).

When measured over time, the two workload measurements (D and 4) should be consistent. In this way, the estimated nurses' workload can be validated. This will be performed by determining the correlation between the workload indication and the perceived workload.

We aim to balance nurses' workload without deteriorating patient experiences and nurses' engagement. In the Netherlands, academic hospitals have chosen to use the validated Consumer Quality Index questionnaire to measure patient experiences. ${ }^{41}$ The questionnaire focuses on specific experiences such as whether information was passed on to the patient in a timely manner or whether nurses have sufficient time to answer patients' questions. This specific information gives clear direction to healthcare providers for improving their processes. We will perform a baseline measurement of patient experiences before we start the time study. After we have developed the aforementioned workload management method and completed implementation, we will do a repeat measurement of patient experiences to see whether controlling the workload of nurses influences patient experiences. We expect to find a non-linear relation, shaped like an example shown in figure 2.

After development and implementation of the workload management method, we will do a follow-up measurement to determine effects on nurses' engagement as well as patient experiences.

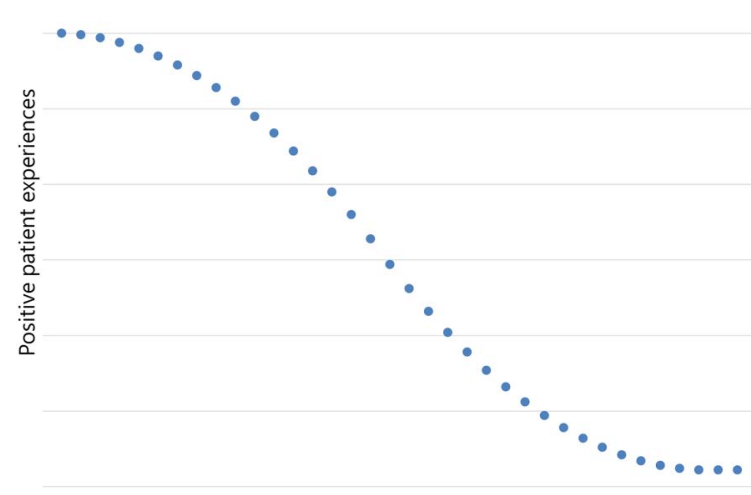

Nurses' workload

Figure 2 Example of expected relationship between positive patient experiences and nurses' workload.

\section{Ethical considerations}

The study will guarantee the privacy of participating patients and staff. Only the lead researcher has access to the master data. Data will be processed in such a way that nothing can be traced back to specific persons.

\section{DISCUSSION}

With this study, we aim to add several new dimensions to nurses' workload management methods. This method will be developed in close cooperation with participating staff nurses and ward management; the strong involvement of the end users of the method will contribute to the usefulness of the method and a broader support of the results. We expect that the method we will develop may also be useful for planning purposes: this is a strong advantage over existing methods, which tend to focus on retrospective analysis. Also, we will analyse data using a mixed model to correct for multilevel data, where usually this is ignored and data are analysed using simple

Table 4 Workload management method (fictitious example)

\begin{tabular}{|c|c|c|c|c|}
\hline Ward $X Y Z$ & June 4 & June 5 & June 6 & \\
\hline Care time for direct patient care (hours) & 46 & 39 & 46 & \\
\hline Time for indirect patient care and additional tasks (hours) & 48 & 40 & 48 & \\
\hline Outcome C: total required care time (hours) & 94 & 79 & 94 & \\
\hline Nurse qualifications & Proficiency & $\begin{array}{l}\text { Allocated care } \\
\text { time (hours) }\end{array}$ & $\begin{array}{l}\text { Allocated care } \\
\text { time (hours) }\end{array}$ & $\begin{array}{l}\text { Allocated care } \\
\text { time (hours) }\end{array}$ \\
\hline Registered nurse $\geq 1$ year experience & $100 \%$ & 10 & 8 & 9 \\
\hline Registered nurse $<1$ year experience & $85 \%$ & 0 & 1 & 1 \\
\hline Student nurse senior & $70 \%$ & 2 & 0 & 2 \\
\hline Student nurse junior & $45 \%$ & 1 & 0 & 0 \\
\hline Outcome 3: total allocated care time (hours) & & 95 & 71 & 90 \\
\hline \multicolumn{2}{|l|}{$\begin{array}{l}\text { Outcome D: expected per cent overstaffing or understaffing } \\
\text { (workload indication) }\end{array}$} & $1 \%$ & $-11 \%$ & $-4 \%$ \\
\hline \multicolumn{2}{|l|}{ Outcome 4: average perceived workload (scale of 1-5) } & 3,1 & 4,2 & 3,2 \\
\hline
\end{tabular}


regression. In our study, we introduce nurses' proficiency as a new dimension in determining workload.

Our study is set in one academic hospital (six wards), UMC Utrecht. It is unclear whether study results can be readily applied to different settings, such as general hospitals. Patient characteristics in general hospitals may be different from characteristics of patients in academic hospitals (since patients with comorbidity or complicated illness are usually referred to academic hospitals, some characteristics are more likely to occur in patients in an academic hospital than in a general hospital). This should not be a problem when applying the study results to general hospitals, since we expect that the biggest difference will be in the frequency of occurrence of characteristics, and not in the types of characteristics or their effect on workload.

Further, the study is set in six surgical wards of six different specialties: this means that when applying the results to other specialties, adjustments will need to be made. Nurses on internal medicine wards spend their time on different activities than nurses on surgical wards. For example, wound care is not expected to be a predominant activity, but nurses are likely to spend a lot of time on, for example, blood transfusions, dialysis or chemotherapy. Different specialties have different working processes, so our study results can be most easily applied to surgical wards. In addition, working processes, organisational structure and outside influences (new laws or protocols, IT developments, etc) may require adjustment to the workload management method.

However, we expect that the framework of the workload management method can be applied in any hospital: it would result in different checklists of patient characteristics and work sampling results, however. We aim to develop a method that is generally applicable or can be modified easily for different hospital settings, specialties or even different types of healthcare providers and that is robust to organisational and process changes.

At UMC Utrecht, hospital management will use this tool to ensure a better balance between patient needs and nursing staff size and expertise. At the moment, we cannot objectively determine whether our nursing capacity is optimally matched to the hospital wards. Nurse management indicates that there are signals that our nurses believe some wards have a much higher workload than others. We aim for a fair and sensible distribution of nursing staff over the wards, resulting in an equally distributed and manageable workload for all nursing staff.

The data collected during the work sampling study are also very interesting from an operational excellence perspective. When further analysed, these data can give valuable insight into the working processes of different wards and can help compare operational excellence between wards and explain differences.

Contributors WFJMvd0 is the corresponding author and guarantor. WFJMvdO and WG conceived the study. WFJMvdO, WG, WR, RKS and HFvS participated in its design. WFJMvdO gathered and cleaned the data, coordinated the study and drafted this manuscript. RKS performed statistical analysis. HFvS helped to draft the manuscript. All authors revised the manuscript drafts and approved the final manuscript.

Funding This study is entirely funded by the University Medical Center (UMC) Utrecht.

Competing interests None declared.

Ethics approval The study protocol was submitted to the medical ethical review board of UMC Utrecht and received a positive advice, protocol number $14-165 / C$.

Provenance and peer review Not commissioned; externally peer reviewed.

Data sharing statement The statistical code and data set are available from the corresponding author.

Open Access This is an Open Access article distributed in accordance with the Creative Commons Attribution Non Commercial (CC BY-NC 4.0) license, which permits others to distribute, remix, adapt, build upon this work noncommercially, and license their derivative works on different terms, provided the original work is properly cited and the use is non-commercial. See: http:// creativecommons.org/licenses/by-nc/4.0/

\section{REFERENCES}

1. Aiken LH, Sloane DM, Bruyneel L. Nursing staff and education and hospital mortality in nine European countries: a retrospective observational study. Lancet 2014;383:1824-30.

2. Liu LF, Lee S, Chia PF, et al. Exploring the association between nurse workload and nurse-sensitive patient safety outcome indicators. J Nurs Res 2012;20:300-9.

3. Unruh L. Nurse staffing and patient, nurse, and financial outcomes Am J Nurs 2008;108:62-71. quiz 72.

4. Hinno S, Partanen P, Vehviläinen-Julkunen K. Nursing activities, nurse staffing and adverse patient outcomes as perceived by hospital nurses. J Clin Nurs 2012;21:1584-93.

5. Van Bogaert $\mathrm{P}$, Clarke S, Willems R, et al. Staff engagement as a target for managing work environments in psychiatric hospitals: implications for workforce stability and quality of care. J Clin Nurs 2013;22:1717-28.

6. Spence Laschinger HK, Grau AL, Finegan J, et al. Predictors of new graduate nurses' workspace well-being: testing the job demands-resources model. Health Care Manage Rev 2012;37:175-86.

7. Ohue T, Moriyama M, Nakaya T. Examination of a cognitive model of stress, burnout, and intention to resign for Japanese nurses. Jpn J Nurs Sci 2011;8:76-86.

8. Mudaly P, Nkosi ZZ. Factors influencing nurse absenteeism in a general hospital in Durban, South Africa. J Nurs Manag 2015;23:623-31.

9. Bakker AB, DE Verbeke W. Using the Job Demands-Resources model to predict burnout and performance. Human Resource Management 2004;43:83-104.

10. Toh SG, Ang E, Devi MK. Systematic review on the relationship between the nursing shortage and job satisfaction, stress and burnout levels among nurses in oncology/haematology settings. Int $J$ Evid Based Healthc 2012;10:126-41.

11. European Commission. Second Biennial Report on social services of general interest. In: Directorate-General for Employment Social Affairs and Inclusion. eds. 2011:9-11.

12. Lacey SR, Cox KS, Lorfing KC, et al. Nursing support, workload, and intent to stay in Magnet, Magnet-aspiring, and non-Magnet hospitals. J Nurs Adm 2007;37:199-205

13. Leone C, Bruyneel L, Anderson JE, et al. Work environment issues and intention-to-leave in Portuguese nurses: a cross-sectional study. Health Policy 2015;119:1584-92.

14. Van Bogaert $P$, van Heusden $D$, Timmermans $O$, et al. Nurse work engagement impacts job outcome and nurse-assessed quality of care: model testing with nurse practice environment and nurse work characteristics as predictors. Front Psychol 2014;5:1261.

15. Twigg D, Duffield C. A review of workload measures: a context for a new staffing methodology in Western Australia. Int J Nurs Stud 2009;46:131-9.

16. Myny D, Van Goubergen D, Gobert M, et al. Non-direct patient care factors influencing nursing workload: a review of the literature. $J A d v$ Nurs 2011;67:2109-29.

17. Holden RJ, Scanlon MC, Patel NR, et al. A human factors framework and study of the effect of nursing workload on patient 
safety and employee quality of working life. BMJ Qual Saf 2011;20:15-24.

18. Upenieks VV, Kotlerman J, Akhavan J, et al. Assessing nursing staffing ratios: variability in workload intensity. Policy Polit Nurs Pract 2007;8:7-19

19. Mueller M, Lohmann S, Strobl R, et al. Patients' functioning as predictor of nurses' workload in acute hospital units providing rehabilitation care: a multi-center cohort study. BMC Health Serv Res 2010;10:295.

20. Van den Heede K, Diya L, Lesaffre $\mathrm{E}$, et al. Benchmarking nurse staffing levels: the development of a nationwide feedback tool. J Adv Nurs 2008;63:607-18.

21. Sermeus W, Delesie L, Van den Heede K, et al. Measuring the intensity of nursing care: making use of the Belgian Nursing Minimum Data Set. Int J Nurs Stud 2008;45:1011-21.

22. Myny D, Van Hecke A, De Bacquer D, et al. Determining a set of measurable and relevant factors affecting nursing workload in the acute care hospital setting: a cross-sectional study. Int J Nurs Stud 2012;49:427-36.

23. Hughes RG, Bobay KL, Jolly NA, et al. Comparison of nurse staffing based on changes in unit-level workload associated with patient churn. J Nurs Manag 2015;23:390-400.

24. Rauhala A, Fagerström L. Determining optimal nursing intensity: the RAFAELA method. J Adv Nurs 2004;45:351-9.

25. Fagerstrom L, Rainio AK, Rauhala A, et al. Validation of a new method for patient classification, the Oulu patient classification $J$ Adv Nurs 2000;31:481-90.

26. Morris $\mathrm{R}$, MacNeela $\mathrm{P}, \mathrm{Scott} \mathrm{A}$, et al. Reconsidering the conceptualization of nursing workload: literature review. J Adv Nurs 2007;57:463-71.

27. Hoi SY, Ismail N, Ong LC, et al. Determining nurse staffing needs: the workload intensity measurement system. J Nurs Manag 2010;18:44-53.

28. Tourangeau A. Nursing skill mix and experience reduce patient mortality. Hosp Q 2002;5:19-20.

29. Duffield C, Roche M, Merrick ET. Methods of measuring nursing workload in Australia. Collegian 2006;13:16-22.
30. Grunveld JE, Leenders JJT, Van der Helm HA. Draaiboek werklastonderzoek in algemene ziekenhuizen. 1988.

31. Keeney S. The Delphi technique in nursing and health research, vol. 1: Wiley-Blackwell, 2011.

32. Pelletier D, Duffield C. Work sampling: valuable methodology to define nursing practice patterns. Nurs Health Sci 2003;5:31-8.

33. Ampt A, Westbrook J, Creswick N, et al. A comparison of self-reported and observational work sampling techniques for measuring time in nursing tasks. J Health Serv Res Policy 2007;12:18-24.

34. Sittig DF. Work-sampling: a statistical approach to evaluation of the effect of computers on work patterns in healthcare. Methods Inf Med 1993;32:167-74.

35. Streiner LD, Norman GR. Health measurements scales: a practical guide to their development and use. Oxford University Press 2008

36. Hedeker D, Gibbons RD. Longitudinal data analysis. 1st edn. Wiley-Interscience, 2006.

37. McHugh MD, Lake ET. Understanding clinical expertise: nurse education, experience, and the hospital context. Res Nurs Health 2010;33:276-87.

38. Schaufeli WB, Bakker AB, Van Rhenen W. How changes in job demands and resources predict burnout, work engagement, and sickness absenteeism. J Organ Behav 2009;30:893-917.

39. van Veldhoven M, de Jonge J, Broersen S, et al. Specific relationships between psychosocial job conditions and job-related stress: a three-level analytic approach. Work Stress 2002;16:207-28.

40. Van Veldhoven M, Meijman TF. Het meten van psychosociale arbeidsbelasting met een vragenlijst: de Vragenlijst Beleving en Beoordeling van de Arbeid [The measurement of psychosocial job demands with a questionnaire: the questionnaire on perception and evaluation of the work]. In: Arbeidsomstandigheden Nlv. eds. Amsterdam. 1994:55-59.

41. Delnoij DM, Rademakers JJ, Groenewegen PP. The Dutch consumer quality index: an example of stakeholder involvement in indicator development. BMC Health Serv Res 2010;10:88. 